\title{
Survey of Long-Term Sequelae in Survivors of a Malignant Hyperthermia Reaction
}

\author{
Kristian Werneid1, Barbara Brandom² \\ ${ }^{1}$ Department of Anesthesiology, University of Pittsburgh Medical Center, Pittsburgh, PA, USA \\ ${ }^{2}$ Department of Anesthesiology, Children's Hospital of Pittsburgh, Pittsburgh, PA, USA \\ Email: kwerneid@yahoo.com, bwb131252@gmail.com
}

Received 7 December 2015; accepted 18 January 2016; published 21 January 2016

Copyright (C) 2016 by authors and Scientific Research Publishing Inc.

This work is licensed under the Creative Commons Attribution-NonCommercial International License (CC

BY-NC).

http://creativecommons.org/licenses/by-nc/4.0/

(c) (i) (9) Open Access

\section{Abstract}

BACKGROUND: Malignant Hyperthermia (MH) is a potentially fatal, autosomal dominant disorder associated with administration of volatile anesthetics and/or the depolarizing paralytic succinylcholine. Symptoms include muscle rigidity, tachycardia, elevated body temperature, and metabolic acidosis, which are secondary to accelerated skeletal muscle metabolism. MH susceptibility can be a chronic condition, and some MH susceptible patients may develop symptoms subsequent to anesthetic exposure. OBJECTIVE: This is the first study examining the sequelae of an MH event after hospital discharge. METHODS: A survey was sent to patients who voluntarily registered with the North American Malignant Hyperthermia Registry, which included questions on severity of symptoms predominating prior to the MH event, one month after the MH event, and presently on a scale of 1 - 10 with a free text option to expound further. Participants were also asked about their opinions on causality between MH and these symptoms. RESULTS: Twenty-three responses were analyzed (34.8\% response rate). Participants were categorized by their age at the time of the MH event and years since the event. Most (83\%) stayed in the ICU between 1 - 4 days, and 39\% experienced the event over 25 years ago. While $43 \%$ did not attribute any long-term symptoms to their MH event, all others believed that certain symptoms were linked, including muscle pain (90\%), muscle cramps (75\%), muscle weakness $(100 \%)$, back/joint pain $(36 \%)$ and depression/anxiety (42\%). CONCLUSIONS: Our study concluded that long-lasting morbidities may be attributed to an MH event. Chronic musculoskeletal symptoms are experienced by the majority of patients who experience acute $\mathrm{MH}$.

\section{Keywords}

Malignant Hyperthermia, Retrospective Study, Complications, Neuromuscular Diseases 


\section{Introduction}

Malignant Hyperthermia (MH) is a potentially fatal, autosomal dominant disorder associated with administration of volatile anesthetics and/or the depolarizing neuromuscular blocker succinylcholine. Symptoms may include muscle rigidity, tachycardia, tachypnea, elevated body temperature, respiratory and metabolic acidosis, which are secondary to accelerated skeletal muscle metabolism [1]. This is not an allergic reaction to anesthetic agents, but rather a response to these agents due to an inherited genetic disorder. Currently, treatment relies on quick diagnosis and prompt dantrolene administration, in addition to supportive care and correction hyperkalemia, cardiac dysrhythmias, hyperthermia, and acidosis.

MH studies thus far have focused on ryanodine receptor mutations, skeletal muscle, and mitochondrial disruption mainly in the animal models. Durham et al. proposed a feed-forward mechanism by which a lower temperature threshold activates the ryanodine receptor type 1 (RyR1) gene in MHS muscle, causing heat stroke, myopathy, and mitochondrial damage [2]. Research by Balog et al. suggests that recovery of MH-susceptible muscle of pigs from tetanic stimulation was significantly slowed as compared to normal muscle tissue [3]. Giulivi et al. demonstrated that increased production of reactive oxygen species, and a decrease in mitochondrial proteins in the presence of $R y R 1$ mutations promote dysregulation of signaling pathways in mice [4].

Emphasis on the episodic anesthetic-induced MH event has obscured the fact that MH susceptibility is a chronic condition, and some MH-susceptible (MHS) patients may experience muscle pain and weakness following exposure to potent inhalation anesthetics. We have yet to determine the long-term clinical implications of an $\mathrm{MH}$ event in humans. This is the first study examining the sequelae of an MH event after hospital discharge.

\section{Materials and Methods}

A survey was sent to patients who voluntarily registered with the North American Malignant Hyperthermia Registry (NAMHR) with approval of the Institutional Review Board (IRB Approval \# PRO13040056). Patients who met all of the following criteria were included: 1) MH episode occurred between 1987 and 2012; 2) received a depolarizing neuromuscular blocker (succinylcholine) or volatile agent (i.e. sevoflurane, desflurane, halothane, etc.); 3) registered with the NAMHR database. In addition, subjects were required to fit into one of two categories: 1) those with confirmed MH susceptibility via contracture test or genetic testing in addition to an MH event; or 2) an MH event was very likely (Clinical Grading Score > 35), but testing was not performed [5]. Exclusion criteria included those who had symptoms similar to $\mathrm{MH}$, but did not receive a depolarizing muscle relaxant and/or volatile agent.

Demographic information included: gender, age at the $\mathrm{MH}$ event (divided into 10-year increments) and age since the MH event (divided into 5-year increments).Questions regarding $\mathrm{MH}$ included: days of intensive care admission following the $\mathrm{MH}$ event, if and how many genetic relatives also experienced an $\mathrm{MH}$ event, and whether or not confirmatory testing of MH susceptibility (contracture or genetic testing) had been performed. There was also a free text option to describe the most vivid events surrounding the event that the subject recalled, and current symptoms they believe are associated with this $\mathrm{MH}$ event.

Check boxes were provided to indicate the following symptoms: headaches, dizziness, weight gain, weight loss, paralysis, numbness, feeling too hot, feeling too cold, muscle pain, muscle cramps, muscle weakness, back pain, joint pain, visual changes, hoarseness, difficulty swallowing, chest pain, chest tightness, leg swelling, acid reflux, frequent urination, difficulty urinating, abnormal bleeding, nausea, vomiting, depression, anxiety, wheezing, difficulty breathing, general illness, abdominal pain, constipation, diarrhea, heart palpitations, and seizures. Once a symptom was affirmed, further questioning included a perception spectrum regarding severity of each symptom on a 1 - 10 scale. These were further subdivided into three distinct periods of time: prior to the MH event, one month after the MH event, and presently. Due to the retrospective nature of this study, the indication of symptom severity at the present time could range from 1 year to 50 years from the actual MH event.

Analysis of results included the calculation of the mean for symptom severity in subjects who responded in the affirmative to a particular symptom. As this remains a purely descriptive survey, analysis of significance was not performed. An example question is shown in Appendix 1.

\section{Results}

A total of 23 responses were collected and analyzed (34.8\% response rate) after three mailings. This retrospective 
analysis included 11 males and 12 females, who were categorized by their age at the time of the MH event: 13\% between 0 - 10 years old, 17\% between 11 - 20, 30\% between 21 - 30, 22\% between 31 - 40, 13\% between 41 50, and 5\% over 50 (Figure 1). Most (83\%) stayed in the ICU between $1-4$ days, and 39\% experienced the event over 25 years ago. In 93\% of respondents, diagnosis of MH was confirmed via caffeine-halothane contracture and/or genetic testing. The remaining 7\% exhibited overwhelming evidence to suggest an $\mathrm{MH}$ event occurred, but either refused to give consent for muscle contracture testing or were unable to do so; however, these three subjects all had causative variants in the RyR1 gene. While $43 \%$ of total respondents did not attribute any long-term symptoms to their MH event, all others believed specific symptoms were linked.

Figure 2(a) and Figure 2(b) demonstrate the twelve most experienced symptoms by our respondents; averages were calculated for each symptom and during each time interval both in graphical and numeric form (prior to the MH event, 1-month after the MH event, and currently). Figure 3 quantifies the respondents who attributed to symptoms to their MH event.

MUSCLE and JOINT-RELATED SYMPTOMS: Of the 23 respondents, muscle pain $(n=10)$, muscle cramps $(n=12)$, muscle weakness $(n=8)$, and back/joint pain $(n=11)$ were the prominent muscular and joint-related symptoms acknowledged. Each of these symptoms increased in severity over time, with the exception of muscle weakness, which increased subsequent to the $\mathrm{MH}$ event, but was comparable in severity over time. Nine out of $10(90 \%)$ attribute their current muscle pain, 9 out of 12 (75\%) attribute muscle cramps, 8 out of 8 (100\%) attribute muscle weakness, and 4 out of 11 (36\%) attribute their back/joint pain to their MH event.

CARDIOPULMONARY SYMPTOMS: Of the 23 respondents, heart palpitations $(n=7)$ and chest pain/ tightness $(n=6)$ were the prominent cardiac/pulmonary symptoms acknowledged. Three out of $7(43 \%)$ attribute their heart palpitations, and 2 out of 6 (33\%) attribute their chest pain and/or tightness to their MH event.

HEADACHES/DIZZINESS: Of the 23 respondents, headaches $(n=15)$ and dizziness $(n=9)$ were prominent

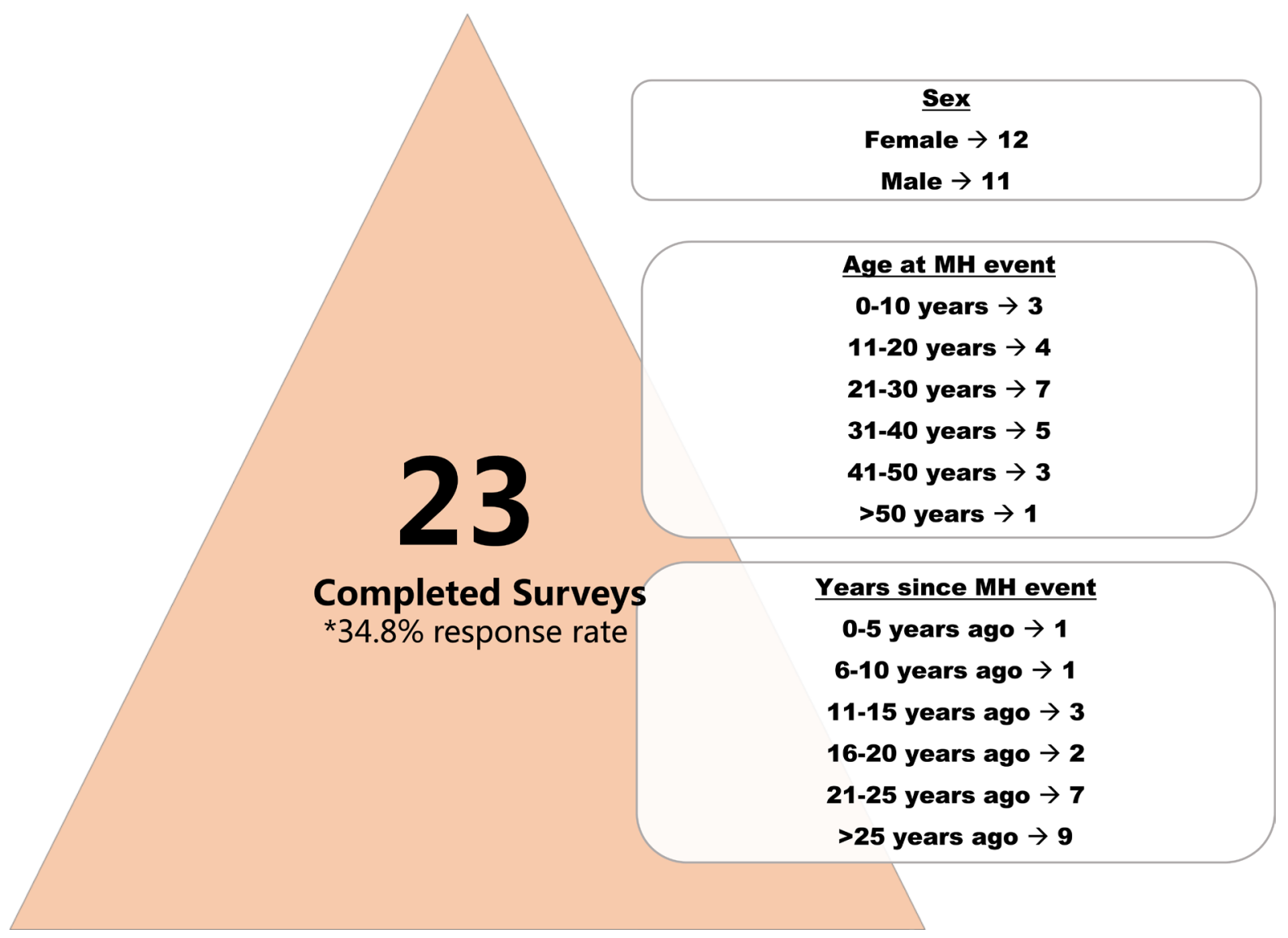

Figure 1. Demographic information of 23 completed surveys, including sex, age at the time of the MH event, and how many years have passed since the MH event. 


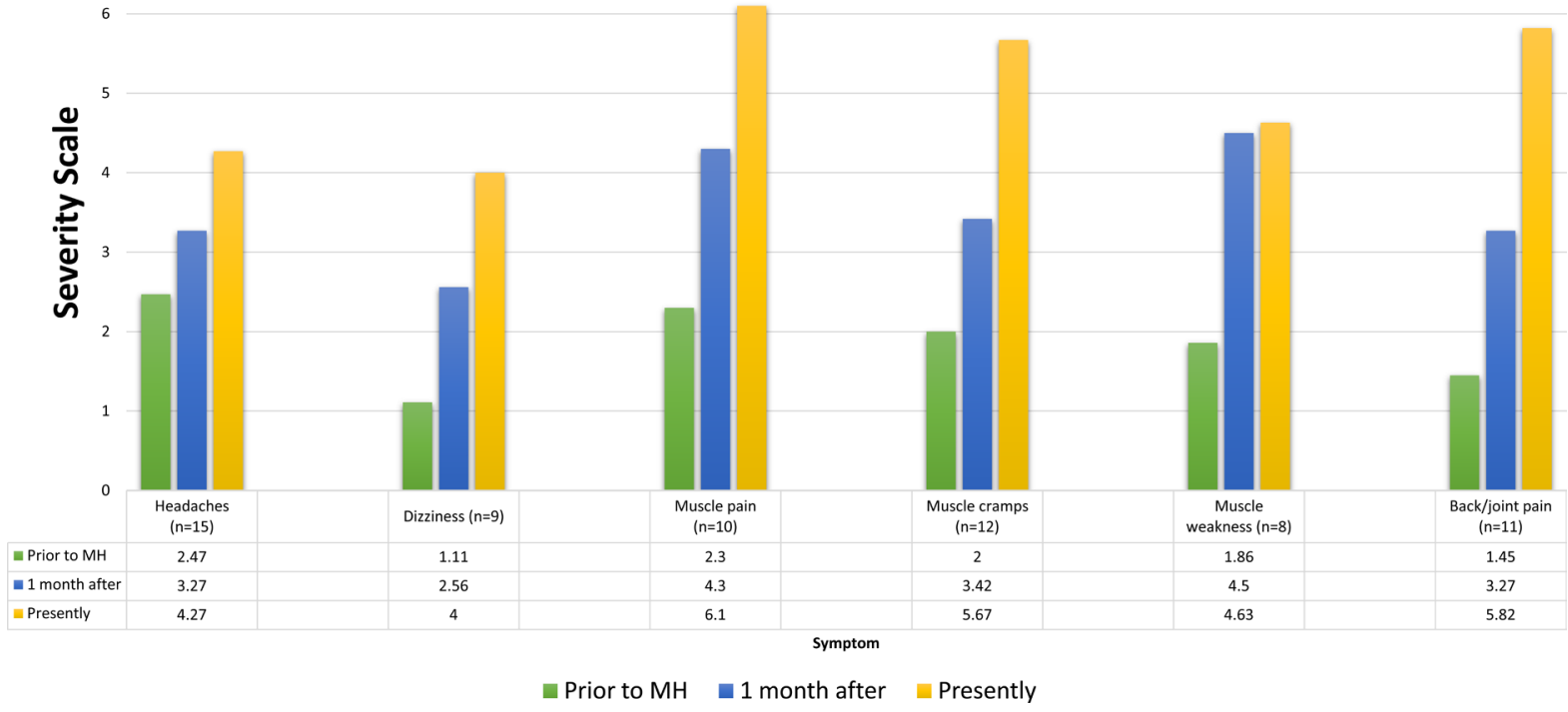

(a)

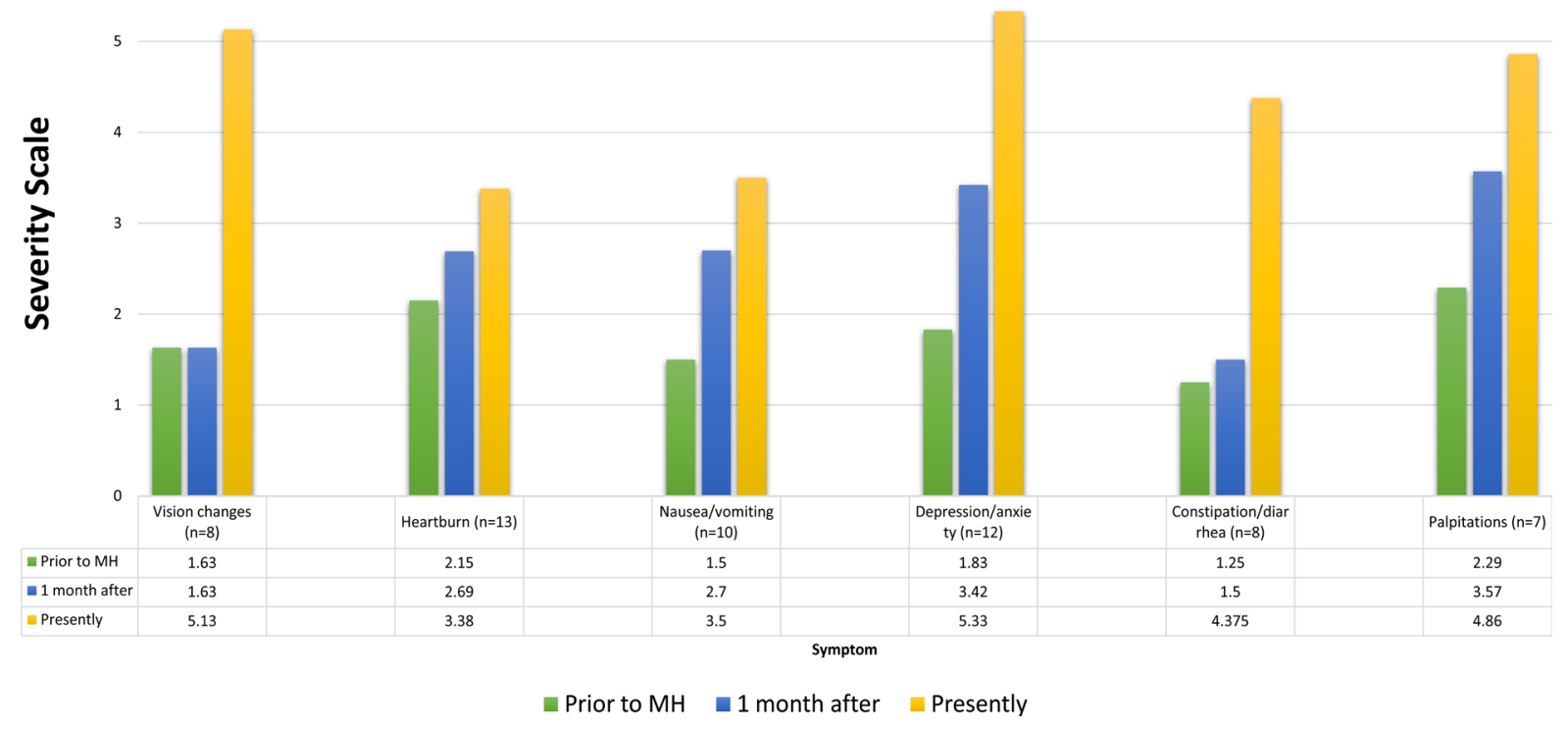

(b)

Figure 2. Symptomatic severity change over time. Each set examines a particular symptom and its change in severity over time (including prior to the MH event, 1 month after the MH event, and severity at the present time). Averages of severity scores were calculated among participants who indicated experience of a particular symptom.

acknowledged subjective symptoms. Three out of 15 (20\%) attribute their headaches, and 2 out of 9 (22\%) attribute their dizziness to the MH event.

GASTROINTESTINAL SYMPTOMS: Of the 23 respondents, diarrhea/constipation $(\mathrm{n}=8)$, nausea/vomiting $(n=10)$, heartburn/acid reflux $(n=13)$ were the prominent GI symptoms acknowledged. Zero out of $11(0 \%)$ attribute their diarrhea and constipation, 2 of 10 (20\%) attribute their nausea and/or vomiting, and 2 of the 13 (15\%) attribute their acid reflux and heartburn to their MH event.

PSYCHIATRIC SYMPTOMS: Of the 23 respondents, anxiety/depression $(n=12)$ were the prominent psychiatric symptoms acknowledged. Five out of 12 (42\%) participants attribute these symptoms to their MH event. 


\section{Symptom attribution to $\mathrm{MH}$ event}

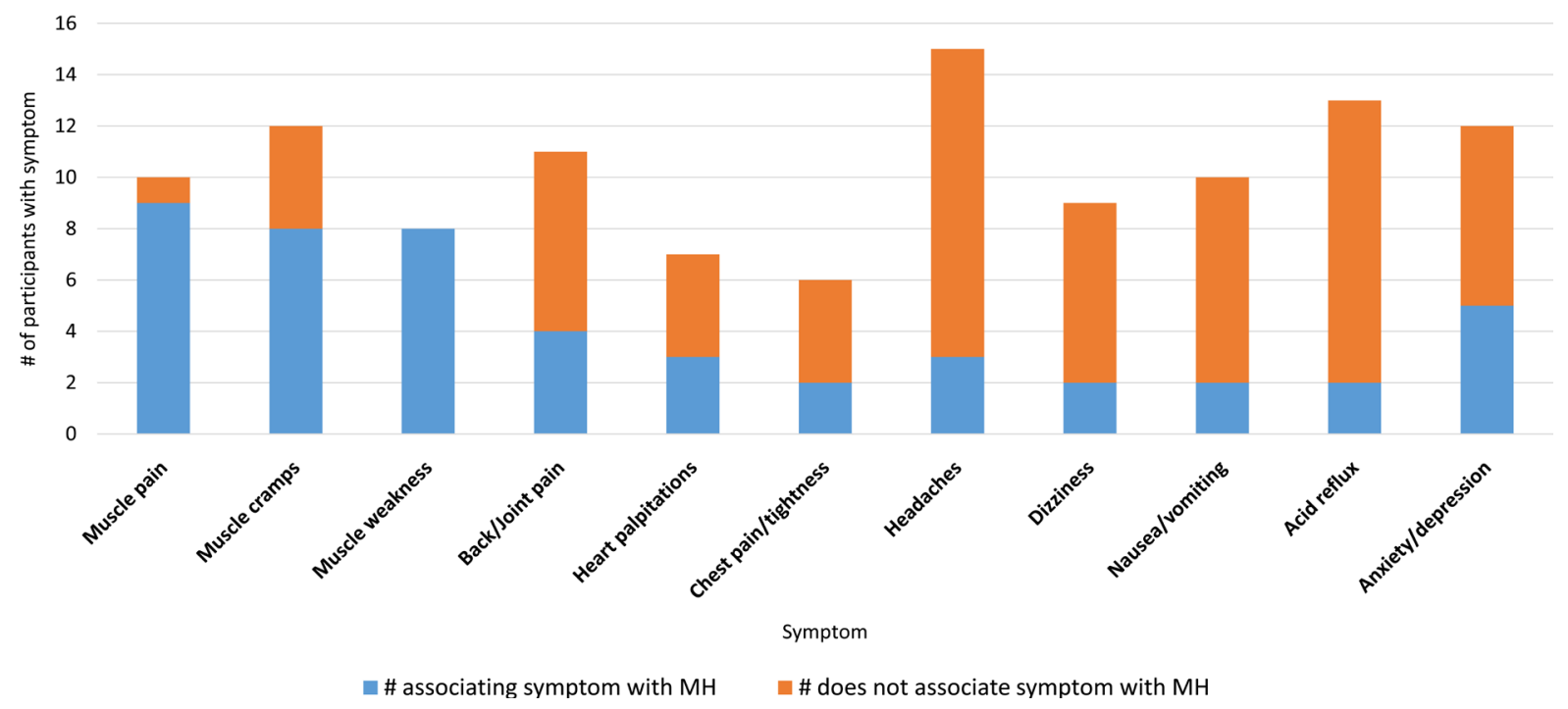

Figure 3. Participants indicating that their MH event resulted in symptoms (blue) and those who did not attribute their symptom to their MH event (orange).

\section{Discussion}

This is the first retrospective, descriptive study examining the long-term effects of an acute anesthetic-induced Malignant Hyperthermia event. Participants from the NAMHR voluntarily provided responses; their recall of symptoms prior to the MH event along with subsequent symptom analysis was reported. There was a substantial increase in musculoskeletal, cardiopulmonary, headache/dizziness, gastrointestinal, and psychiatric symptoms over time after an $\mathrm{MH}$ event. Many participants attributed their muscle pains, muscle cramps, and muscle weakness to their MH event. Fewer participants attributed back/joint pain or depression/anxiety to their MH event. This strongly suggests that the symptoms attributed to musculature were not a result of generalized agerelated malaise and atrophy.

Since injury to skeletal muscle may occur during acute anesthetic-induced $\mathrm{MH}$, these symptoms are not surprising. Andersson et al. focused on the oxidized protein of a RyR1 mutation in rodents, causing calcium leak and leading to increased muscle weakness and decreased exercise capacity with advancing age. These symptoms, however, were improved by stabilizing the RyR1 mutant with calstabin1, suggesting that even in the presence of a RyR1 mutation, there is an element of symptomatic reversibility [6]. Taivassalo et al. studied short-term aerobic training in patients with mitochondrial myopathies, nonmetabolic myopathies and normal subjects, demonstrating over 8 weeks a significant improvement in both oxidative capacity (using phosphorus magnetic resonance spectroscopy) and quality of life in the mitochondrial myopathy group. Although no defined studies to date demonstrate that physical therapy will speed the rate of recovery (as opposed to rest) after an $\mathrm{MH}$ event, long-term physical therapy will likely be a critical component of recovery for these patients [7].

The psychiatric component is critically important, as it speaks to the long-term post-traumatic stress that accompanies such an event. Subsequent treatment would likely involve psychiatric therapy as well.

Two main biases exist within this study, especially with regard to recall and selection. Our data are dependent on accurate memory of symptoms prior to and just after the MH event. Time intervals between the "1-month after your MH event" to "presently" can vary between one and 50 years in this study population. This protocol has no means to assess the accuracy of these memories. In addition, our selection criteria included only those who volunteered to be part of the registry, and who were motivated to return the questionnaire. This may bias our study to those who are symptomatic. Prospective evaluation of a population based cohort might find a lower percentage of subjects with these chronic symptoms. Future studies will require the following analysis: 1) how many experience a given symptom, 2) how symptoms worsen/improve over time and to what degree, and 3) how quickly these symptoms progress or resolve. In addition, an appropriate control group could include family members who tested positive for MHS, but never experienced an MH event themselves. 
Our study concluded that long-lasting morbidities may be attributed to an MH event. In this retrospective survey, chronic muscular symptoms are reported by the majority of patients who experienced acute MH. While causality cannot be determined, the prevalence of muscle pains, cramps, and weakness after an MH crisis warrants further investigation into the long-term post-anesthetic consequences of $\mathrm{MH}$.

\section{Acknowledgements}

The project described was supported by the National Institutes of Health through Grant Numbers UL1 RR024153 and UL1TR000005.

\section{Conflict of Interest}

The authors have no conflict of interest to report.

\section{References}

[1] Larach, M.G., Landis, J.R., Bunn, J.S. and Diaz, M. (1992) Prediction of Malignant Hyperthermia Susceptibility in Low Risk Subjects. An Epidemiologic Investigation of Caffeine Halothane Contracture Responses. The North American Malignant Hyperthermia Registry. Anesthesiology, 76, 16-27. http://dx.doi.org/10.1097/00000542-199201000-00003

[2] Durham, W.J., Aracena-Parks, P., Long, C., et al. (2008) RyR1 S-Nitrosylation Underlies Environmental Heat Stroke and Sudden Death in Y522S RyR1 Knockin Mice. Cell, 133, 53-65. http://dx.doi.org/10.1016/j.cell.2008.02.042

[3] Balog, E.M., Enzmann, N.R. and Gallant, E.M. (2000) Malignant Hyperthermia: Fatigue Characteristics of Skeletal Muscle. Muscle \& Nerve, 23, 223-230. http://dx.doi.org/10.1002/(SICI)1097-4598(200002)23:2<223::AID-MUS13>3.0.CO;2-8

[4] Giulivi, C., Ross-Inta, C., Omanska-Klusek, A., et al. (2011) Basal Bioenergetics Abnormalities in Skeletal Muscle from Ryanodine Receptor Malignant Hyperthermia-Susceptible R163C Knock-in Mice. Journal of Biological Chemistry, 286, 99-113. http://dx.doi.org/10.1074/jbc.M110.153247

[5] Larach, M.G., Localio, A.R., Allen, G.C., et al. (1994) A Clinical Grading Scale to Predict Malignant Hyperthermia Susceptibility. Anesthesiology, 80, 771-779. http://dx.doi.org/10.1097/00000542-199404000-00008

[6] Andersson, D.C., Betzenhauser, M.J., Reiken, S., et al. (2011) Ryanodine Receptor Oxidation Causes Intracellular Leak and Muscle Weakness in Aging. Cell Metabolism, 14, 196-207. http://dx.doi.org/10.1016/j.cmet.2011.05.014

[7] Taivassalo, T., Matthews, P.M., DeStefano, N., et al. (1996) Combined Aerobic Training and Dichloroacetate Improve Exercise Capacity and Indices of Aerobic Metabolism in Muscle Cytochrome Oxidase Deficiency. Neurology, 47, 529534. http://dx.doi.org/10.1212/WNL.47.2.529 


\section{Appendix 1. Sample Question and Answer on REDCap}

MUSCLE PAIN, CRAMPS, WEAKNESS

\begin{tabular}{|c|c|c|c|c|c|c|c|c|c|c|}
\hline & \multicolumn{2}{|c|}{ YES, muscle pain } & \multicolumn{3}{|c|}{ YES, muscle cramps } & \multicolumn{2}{|c|}{$\begin{array}{l}\text { YES, muscle } \\
\text { weakness }\end{array}$} & \multicolumn{3}{|c|}{ NO } \\
\hline $\begin{array}{l}\text { Have you ever experienced } \\
\text { MUSCLE PAIN or MUSCLE } \\
\text { CRAMPS or MUSCLE WEAKNESS? } \\
\text { tmust provide value }\end{array}$ & (14) & 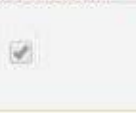 & \multicolumn{3}{|c|}{$\square$} & \multicolumn{2}{|c|}{ Ei } & \multicolumn{3}{|c|}{$\square$} \\
\hline \multicolumn{11}{|c|}{ How would you rate your MUSCLE PAIN: } \\
\hline & 1-mild & 2 & 3 & 4 & $\begin{array}{l}5 . \\
\text { moderate }\end{array}$ & 6 & 7 & 8 & 9 & $\begin{array}{l}10- \\
\text { severe }\end{array}$ \\
\hline $\begin{array}{l}\text { PRIOR to your MH event } \\
\text { must provide value }\end{array}$ & (14) & (1) & (3) & Q & (6) & 0 & (1) & Q & 6) & (11) \\
\hline 1 month AFTER your MH event & (4) & (c) & 3) & Q & Q & Q & (2) & 0 & (e) & 8 \\
\hline $\begin{array}{l}\text { Currently / Now } \\
\text { must provide value }\end{array}$ & (t) & Q & P & 0 & $\Theta$ & (1) & (6) & 0 & (e) & 0 \\
\hline \multicolumn{3}{|c|}{$\begin{array}{l}\text { Comments: Please describe your MUSCLE PAIN in as much } \\
\text { detail as you recall. }\end{array}$} & \multicolumn{8}{|c|}{$\begin{array}{l}\text { Usually occurs when I take multiple days off from my } \\
\text { normal cycling routine that consists of riding } 3-4 \text { times a } \\
\text { week } 30-70 \text { miles at a time with an avg speed of } 18- \\
20 \text { mph. The pain is like someone has a knife in my } \\
\text { thigh and is twisting it. The pain will move to various } \\
\text { places in my upper legs during an episode from inner, } \\
\text { outer, upper, lower quads and glutes. }\end{array}$} \\
\hline
\end{tabular}

